DOI: $10.24850 / \mathrm{j}-$ tyca-2020-06-03

Artículos

\title{
Los estudios del agua en Chile: revisión y perspectivas críticas
}

\section{Water research in Chile: Review and critical perspectives}

Raphael Cantillana1, ORCID: https://orcid.org/0000-0002-8979-4024

1Universitat Autònoma de Barcelona, GRAFO, Barcelona, España, raphael.cantillana@uab.cat

Autor para correspondencia: Raphael Cantillana, raphael.cantillana@uab.cat

\section{Resumen}

Chile, un país que posee algunas de las regiones más áridas del planeta, fue, sin embargo, el primer país del mundo en privatizar sus recursos hídricos. La conjunción de estos dos fenómenos lo convierte en un caso excepcional para la investigación del uso y gestión del agua, interés que se ha visto reflejado en las últimas décadas por el aumento de la producción científica de este campo multidisciplinario. Esta producción ha contribuido al análisis, por una parte, de la política hídrica chilena y las 
lógicas neoliberales que le subyacen, mientras que por otra se ha centrado en los conflictos ambientales y las prácticas indígenas. El presente artículo revisa la literatura en este ámbito del conocimiento, proponiendo un balance histórico y una discusión de los principales temas para finalizar con una reflexión a propósito de los nuevos desafíos para la investigación de este dinámico campo de estudio: el agua, en modo alguno, una realidad física separada de su tejido sociocultural.

Palabras clave: crisis del agua, gestión del agua, recursos hídricos.

\begin{abstract}
Although Chile has some of the most arid regions on the planet, it was the first country in the world to privatize its water resources. The combination of these two phenomena turns it into an exceptional case for the use and management of water research. Thereby, a growing interest in this multidisciplinary field has been lately reflected by an increase in its scientific production. This production has contributed to describe, on the one hand, the Chilean water policy and the neoliberal logics that underlie it, while it has also focused on environmental conflicts and indigenous practices. This paper reviews the literature in this field of knowledge, proposing a historical balance and discussing the main topics researched, to end with a reflection on the new challenges for research in this dynamic field of study: the water, a physical reality never separated from the sociocultural fabric.
\end{abstract}

Keywords: Water crisis, water management, water resources. 
Tecnología y

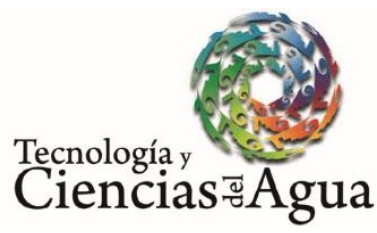

Recibido: 12/12/2019

Aceptado: 02/03/2020
2020, Instituto Mexicano de Tecnología del Agua

Open Access bajo la licencia CCBY-NC-SA 4.0

(https://creativecommons.org/licenses/by-nc-sa/4.0/)

\section{Introducción}

Actualmente, la crisis del agua es una realidad cuya comprensión es exigua sin la atención tanto a su historia política como a los determinantes ambientales. Así, desde la segunda mitad del siglo XX, el acceso al agua $y$ al saneamiento se convirtieron en temas clave en los organismos internacionales, junto con una política de crecimiento económico acelerado de sus estados miembros (e.g., Carta de Punta del Este, 1961; ONU, 1977). Esta coyuntura política inició un proceso depredador de los recursos naturales, con consecuencias ecológicas tales que han llevado a una profunda crisis del agua, especialmente por lo que se refiere a la escasez y sobreexplotación (Arsel, Hogenboom, \& Pellegrini, 2016; International Conference on Water and the Environment, 1992; World Water Council, 2018).

Estas dinámicas operaron en Chile de forma particular, ya que como consecuencia de su condición de laboratorio de políticas neoliberales fueron privatizadas las empresas del Estado y los bienes públicos (Harvey, 2005). De hecho, fue en plena dictadura militar de Pinochet que se 
instauró el Código de Aguas (CA), una política que favorece la comercialización privada de los recursos hídricos, estableciéndolo como un bien nacional de uso público, al mismo tiempo que como un bien privado (Bauer, 2008; Larraín, 2006). Esta dualidad teórica es resuelta en la práctica por el dominio de la mercantilización del agua, génesis de un proceso con dimensiones socioculturales y ambientales devastadoras, que aumentan las condiciones de escasez hídrica, producen daño ambiental, y generan conflictos que intensifican la crisis del agua en Chile (Babidge, 2016; Bauer, 2004; Budds, 2018).

En este contexto, la investigación del agua ha sido abordada desde diferentes perspectivas, mostrando la complejidad de un fenómeno en el que la cultura, la sociedad y el ambiente son elementos en interacción constante. De esta forma, la investigación del agua en Chile revela las dinámicas de la avanzada neoliberal en la política hídrica y los mercados de agua, la sobreexplotación extractiva, el uso y conocimiento local indígena, y una serie de campos en intersección. Temas que han sido investigados desde la sobreexplotación minera en regiones de extrema aridez del Norte Grande (e.g., Babidge, 2016; Prieto, 2016) hasta los conflictos producidos por proyectos hidroeléctricos en la húmeda Patagonia (e.g., Romero-Toledo, Romero-Aravena, \& Toledo, 2009; Torres-Salinas, García, \& Rojas, 2017), entre otros.

A pesar de esta importante producción científica, no existe aún una revisión que permita reconocer los resultados, vacíos, limitaciones y proyecciones que ha presentado la investigación en estos temas. En consecuencia, resulta alentador abordar dicha tarea con el objetivo de establecer el estado de la investigación del agua elaborada en Chile por 
investigadores a nivel global, desde una mirada de las ciencias sociales y humanidades. Para ello, es necesario comenzar con un balance histórico y cuantitativo de las producciones académicas, desde la apertura del debate hasta la actualidad, revisando los investigadores con mayor producción, las unidades de estudio a nivel regional y las metodologías aplicadas. A continuación, se expone un recorrido por los principales temas de investigación, discutiendo sus formas y problemáticas. Por último, hay una reflexión a propósito de los nuevos desafíos para la investigación de este campo de estudio, observando limitaciones y proyecciones.

\section{Materiales y métodos}

A partir de la concepción del agua como un elemento complejo resultante de la interacción entre dimensiones culturales, políticas y ambientales, la selección de las fuentes de información consistió en una elección de keywords que tuvieron en cuenta esa complejidad. Tales palabras clave incluyeron a Chile como una constante, a las que se sumaron conflictos por el agua, agua indígena, gobernanza del agua, políticas hídricas, gestión del agua y ciclo hidrosocial, entre otras. El idioma de búsqueda fue el inglés, entendiendo la norma general en publicaciones indexadas de usar, independiente de la lengua de los documentos, títulos, 
resúmenes y palabras clave en dicho idioma. La búsqueda se efectuó en los motores de Web of Science (WoS), Scopus, Scielo y en la misma bibliografía de los documentos revisados. Con respecto al marco temporal, se realizó una búsqueda histórica desde el año en que se encontraron las primeras investigaciones hasta el 2019, aunque este último, por estar en curso, no se consideró en el balance cuantitativo. A los 821 resultados obtenidos se les aplicó un filtro de revisión que priorizó la producción disciplinar de las ciencias sociales y humanidades, u otras disciplinas cuyas investigaciones abordaran de forma trasversal el fenómeno de estudio (e.g., Lictevout \& Faysse, 2018).

El resultado final arrojó 180 producciones entre artículos, libros, capítulos en libros, actas y reportes; de ellos 105 en inglés y 75 en español, alojados en gestores de referencias bibliográficas y posteriormente procesados en SPSS. Los datos se codificaron por año, autor principal, tipo de documento, indexación, fuente de información, metodología de investigación, región de investigación; y, por último, se agruparon en seis temas principales. Esta decisión final es para facilitar la descripción y discusión de los tránsitos investigativos. Con respecto de estas disposiciones metodológicas, existen dos obstáculos: el primero de ellos se produjo al clasificar en un nivel de agregación mínima los casos de estudio, habiendo investigaciones que referían a más de uno, por lo que se decidió su clasificación a nivel regional; el segundo problema surgió al agrupar los estudios por temas principales, puesto que se trató un objeto de estudio transversal con diversos temas en yuxtaposición. Por ejemplo, las investigaciones sobre el conflicto o el extractivismo de igual forma poseen un análisis de la política hídrica, situación en la que el objetivo descrito en cada documento se consideró como prioridad. 


\section{Balance de los estudios del agua en Chile}

En 1985 comenzó el debate sobre el uso y la gestión del agua en Chile, junto con investigadores que situaron su interés en la desposesión producida por el nuevo CA en los pueblos indígenas del norte (e.g., Aldunate, 1985; Van Kessel, 1985). A este debate iniciado por la antropología, se integraron disciplinas como la sociología (Guerrero, 1991), geografía (Bauer, 1993) y economía (Alé-Yarad, 1990), centrándose en las descripciones históricas del sector hídrico, en las dinámicas de los mercados de agua y la gestión del Estado. Durante la década del 2000, la cantidad de contribuciones aumenta a casi cinco por año, con un enfoque acentuando en las consecuencias negativas del CA. Algunos (Budds, 2004a), desde la ecología política (EP), ya observan las relaciones de poder que subyacen en las disputas por el recurso hídrico. Hacia la década actual (2010), se produce un aumento de las publicaciones con casi 14 contribuciones anuales en promedio, las cuales adoptan un enfoque multidisciplinario. Estas investigaciones adoptan una posición crítica y defensiva en relación con la política hídrica, que se convierte, al mismo tiempo, en un elemento común. Durante esta misma anualidad, también surgen estudios que incorporan el análisis de la agenda extractivista y los conflictos por el agua (e.g., Babidge \& Bolados, 
Tecnología y

Ciencias $₫$ Agua
2020, Instituto Mexicano de Tecnología del Agua

Open Access bajo la licencia CC BY-NC-SA 4.0

(https://creativecommons.org/licenses/by-nc-sa/4.0/)

2018; Prieto, Salazar, \& Valenzuela, 2019). Esta distribución histórica puede observarse en la Figura 1.

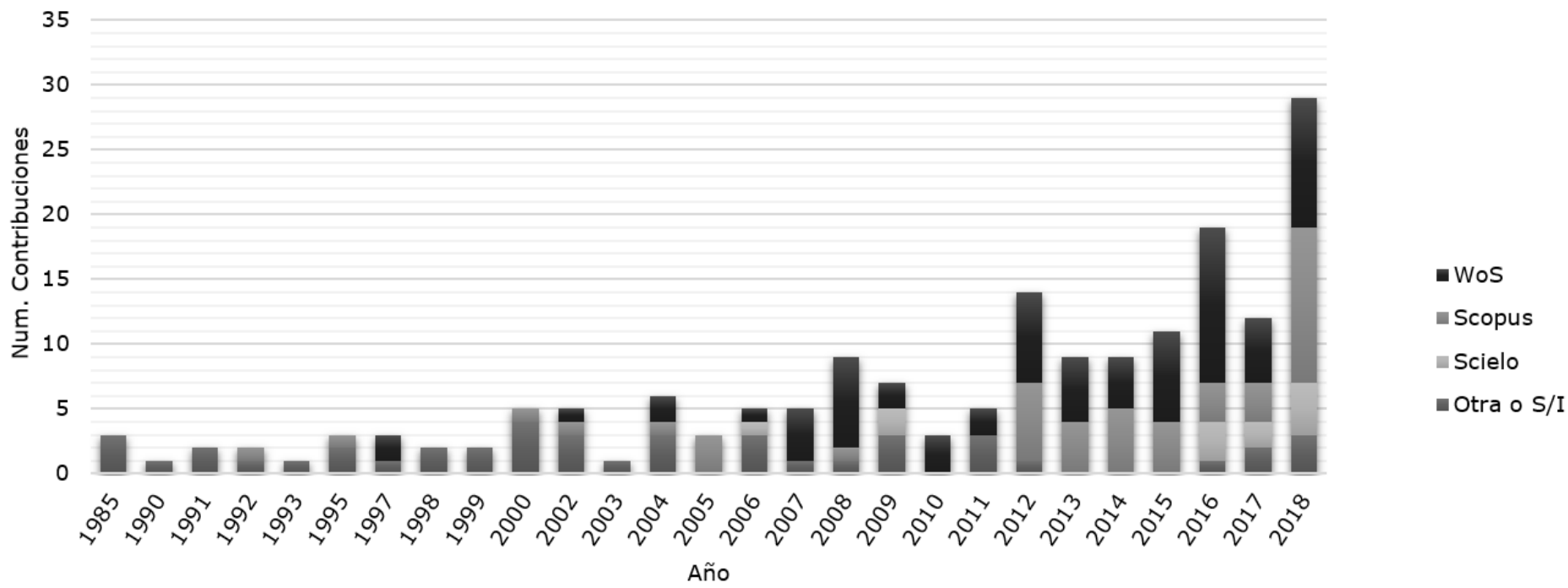

Figura 1. Distribución de la literatura relacionada con el agua en Chile por años y tipo de indización. Fuente: elaboración propia.

Por otra parte, las investigaciones examinan distintas realidades con casos en 13 regiones del país. Por ejemplo, la Figura 2 muestra el área de estudio con mayor número de contribuciones, que es el Norte Grande de Chile. No es casual la concentración en esta área que posee particularidades para la investigación del agua: el desierto de Atacama es uno de los puntos más áridos del planeta, existen prácticas de uso y gestión ancestral indígena y, además, en esta zona se sitúan los megaproyectos de extracción minera que demandan altas cantidades de agua (e.g., Gentes, 2003; Prieto, 2015; Carrasco, 2016). No obstante, de las 180 investigaciones revisadas, 86 de ellas se centraron en Chile como 
caso de estudio general. En el ámbito nacional se ubican algunos análisis de la política hídrica o estudios del sector hidráulico nacional (e.g., Baer, 2014; Celedon \& Alegría, 2004; Hurlbert \& Diaz, 2013).

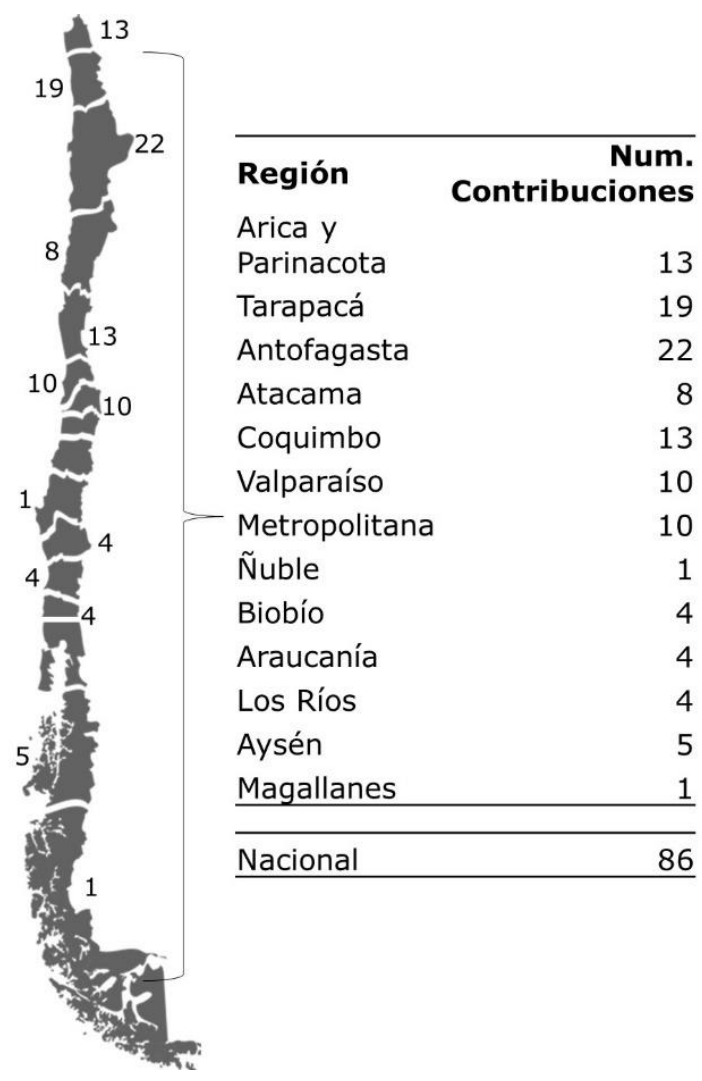

Figura 2. Distribución de contribuciones por área de estudio en los ámbitos regional y nacional. Fuente: elaboración propia.

A pesar del aumento progresivo de la producción científica en este campo, resaltan seis autores principales con cinco o más contribuciones, variación visible en la Figura 3 (e.g., Bauer \& Catalán, 2017; Budds, 2018; Gentes, 2006; Prieto et al., 2019; Torres-Salinas \& Rojas, 2018). Sus 
estudios, desde distintas disciplinas, responden a un enfoque que pone la mirada en los pueblos indígenas, los conflictos ambientales y las dinámicas de los mercados de agua. Otro dato relevante surge al analizar el tipo de fuente de información empleado en el total de las investigaciones, en la cual existe una preponderancia de un $51 \%$ por el uso de fuentes secundarias por sobre las primarias $(12.8 \%)$ y mixtas (35.6\%). Un panorama más tangencial recibe las investigaciones cualitativas, con sólo 56 de 180 contribuciones que refieren al uso de esta metodología.

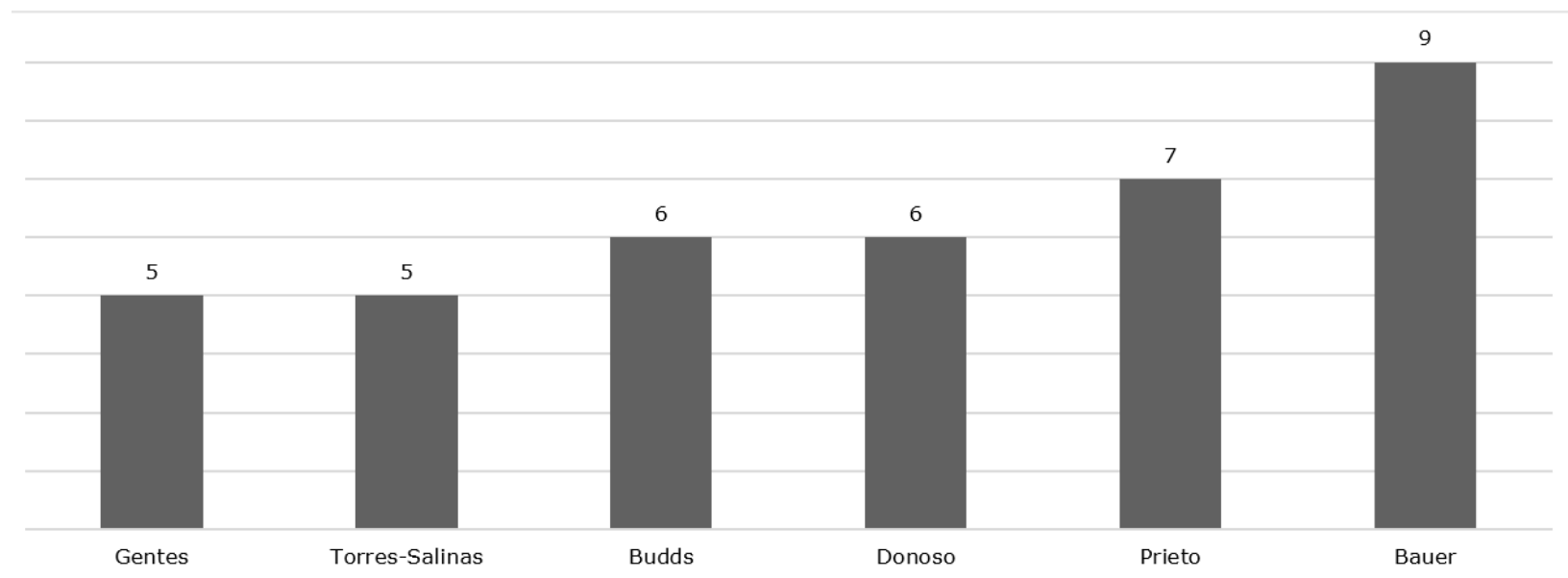

Figura 3. Autores con mayor número de contribuciones. Fuente: elaboración propia.

\section{Temas centrales en la investigación del agua}


Recapitulando los resultados presentados, las investigaciones del agua en Chile otorgan un efectivo examen multidisciplinario con diversas apreciaciones a las conexiones de su uso y gestión. A pesar de esta gama y de la interacción propia de los tópicos, en la discusión sucesiva se procura agrupar este recorrido en los siguientes seis temas principales.

\section{Análisis de la política hídrica y el Código de Aguas}

El surgimiento de la investigación científica del agua en Chile estuvo marcado por la entrada en vigor del CA. Razón por la que este aparato de la política hídrica nacional ha estado presente en casi la totalidad de las contribuciones, con posiciones a favor o en contra. Así, el CA ha quedado establecido como un instrumento del Estado, cuya concepción no es otra que fundar el agua como un recurso explotable al servicio del crecimiento económico (Bauer, 2013; Boelens \& Vos, 2012; Larraín, 2006). Por ejemplo, para Domper (2006), la política hídrica con ideología de mercado ha sido efectiva y modernizadora para el Estado, el cual, paradójicamente, según Donoso (2015), ha tenido una débil gestión institucional. Ambas aportaciones se hacen desde una concepción mercantil del agua. 
Aparte de estos argumentos, no es sino hasta la aparición del trabajo de Bauer, Contra la Corriente (1998), que la investigación relacionada con la ley de agua se desplaza hacia perspectivas que consideran las dimensiones humanas. Bauer demostró cómo la realización de un trabajo de campo in situ puede aportar datos que hasta el momento permanecían olvidados en la literatura. Su investigación podría considerarse como una de las primeras problematizaciones complejas de los impactos del mercado del agua en Chile. Las lecciones que a posteriori ofrece Bauer (Bauer, 2004; Bauer, 2005; Bauer, 2008; Bauer, 2013) exponen la desmitificación del modelo chileno en el debate internacional. Tanto es así que Solanes (2013) evidencia el infructífero intento de emulación de la normativa chilena en el Perú. Otras investigaciones permiten sostener que las reformas del CA no suprimieron su imposición top-down ni consideraron la participación de las comunidades involucradas (Gentes, 2006; Hurlbert \& Gupta, 2015). Es más, la idea de una gestión integrada habría quedado plasmada de forma discursiva y cortoplacista (Hill, 2013; Kretshmer, Wendt, \& Oyarzún, 2012). Da la impresión de que, para estos autores, la experiencia chilena constituińa un cúmulo de enseñanzas sobre acciones que no se deberían aplicar para una correcta gestión gubernamental del agua.

Budds (Budds, 2009; Budds, 2013; Budds, 2018) también ofrece otras contribuciones empíricas con un enfoque desde la EP que examina las relaciones de poder que subyacen en la gestión local del agua en el río La Ligua. El caso denota la estrecha conexión entre un neoliberalismo altamente institucionalizado y la gestión mercantil del agua, condiciones que consolidarían las relaciones de poder (Budds, 2013). Otra de sus críticas las dirige al dominio del conocimiento físico y natural en la gestión 
gubernamental, situación donde predominaría el ciclo hidrogeológico por delante de otras consideraciones sociales y políticas (Budds, 2009). Ante esto, Budds propone la necesidad de avanzar hacia un modelo hidrosocial que reconozca las relaciones socioculturales en la gestión local. La importancia de este modelo analítico yace en su precisión para desentrañar las relaciones dialécticas producidas a escala colectiva e individual entre las personas y su entorno natural (Linton \& Budds, 2014).

En otras contribuciones se estudian las consecuencias de la política hídrica para los pueblos indígenas. Para el norte de Chile, Gentes (Gentes, 2002; Gentes, 2003) ha trabajado empírica y documentalmente los problemas que derivan de la incapacidad del Estado por otorgar un reconocimiento constitucional a los indígenas, ignorando su relevancia cultural y política. A nivel más general, éste y otros autores sostienen que, a pesar de que algunos Estados han avanzado en algún nivel el reconocimiento de los grupos étnicos, su derecho ancestral al agua y a la gestión del territorio ha sido ocultado o negado en las políticas hídricas (Boelens et al., 2012; Gentes, 2002). Incluso, es posible sostenerque las consecuencias de este tipo de leyes destruyen cualquier ánimo reparatorio de los abusos sistemáticos hacia los pueblos indígenas en Chile (Lovera, 2017), ignorando no sólo sus prácticas culturales, sino también su territorio ancestral (Madaleno \& Gurovich, 2007). Estas contribuciones acerca de las consecuencias en los pueblos indígenas no se agotan aquí, sino que también colisionan con otros temas que serán abordados más adelante. 
Tecnología y

Ciencias $₫$ Agua
2020, Instituto Mexicano de Tecnología del Agua

Open Access bajo la licencia CC BY-NC-SA 4.0

(https://creativecommons.org/licenses/by-nc-sa/4.0/)

\section{Mercados de agua}

Los mercados de agua, como espacios donde se practica la eventual libre transacción de derechos de agua, constituyen el espíritu del CA y sus reformas (Congreso Nacional de Chile, 1981). En ellos se reconocen dos tratamientos relativos. Uno de los ámbitos observa la forma en que opera la transacción de los derechos de agua y otra se centra en la utilización de los mercados de agua como etiqueta para la dinámica propia de la mercantilización. Con respecto al primer tratamiento, las lecturas inaugurales en este tema revelan la mitificación empresarial consensuada de la valorización monetaria del agua (e.g., Gazmuri, 1992; Ríos \& Quiroz, 1995). Ante todo, esta primera evaluación señala, paradójicamente, que las transacciones han fomentado el uso agrícola de forma eficiente (Gazmuri, 1992). De hecho, para Thobani (1997), los mercados han ayudado a la sociedad y al Estado, circunstancia que a la vista de Donoso (2013) debiera asegurar el éxito a través de una efectiva asignación del recurso.

Las contribuciones críticas de Bauer (1997) y Budds (2004b) defienden que los mercados del agua han enfrentado, entre otros, dos problemas iniciales: (1) no se ha establecido una lista de precios informados, impidiendo (2) la capacidad de establecer un libre mercado, cuyo resultado es el acaparamiento de los derechos de explotación. Por otra parte, la concentración de la asignación del recurso ha facilitado la producción de una agricultura de productos para la exportación, lo que 
tiene consecuencias para las pequeñas explotaciones. En este sentido, lo que ocurre con los pequeños agricultores puede entenderse mejor a través del análisis distributivo de estos derechos en la cuenca del Limań o el río La Ligua, donde este grupo vulnerable habría sido desplazado y marginado (Budds, 2004b; Romano \& Leporati, 2002). Al comparar estos alegatos con otras investigaciones con conclusiones positivas al modelo transaccional, surgen algunas disrupciones en estas últimas. Por ejemplo, no se profundizan las características particulares de los agricultores o granjeros como sujetos de estudio, evidenciando así, dificultades ontológicas importantes al no considerar las lógicas productivas de estos grupos humanos con la industria (e.g., Grafton, Libecap, McGlennon, Landry, \& O'Brien, 2011; Hearne \& Donoso, 2014; Molinos-Senante, Donoso, \& Sala-Garrido, 2016).

En otro orden de ideas, las dinámicas de los mercados de agua no sólo alcanzan la agricultura, sino también la producción de la gran minería. Los fenómenos que aquí suceden han sido particularmente observados desde los métodos cualitativos con enfoques teóricos desde la EP. En principio, la posibilidad de incorporar al patrimonio privado los derechos de explotación del agua permitió, en el caso del norte del país, la inscripción de derechos particulares que marginaron a las comunidades indígenas (Budds, 2010). Especialmente, en el caso de los atacameño, Prieto (Prieto, 2015; Prieto, 2016) ilustra las dimensiones de la desposesión y desmitifica el espíritu laissez faire de la lógica neoliberal. Este análisis se sustenta en las complejas instituciones de un Estado que, en la práctica y para el caso de estudio, no permite una libre competencia, reservándose éste un rol fundamental en la asignación del recurso. Otra de las contradicciones existentes abonan a la creencia de que la 
mercantilización y la valoración monetaria del agua evitan un despilfarro, ya que, contrariamente, la asignación del agua para uso minero es abundante en comparación con el uso domiciliario (Bitran, Rivera, \& Villena, 2014; Bottaro, Latta, \& Sola, 2014). Algunas de éstas y otras contradicciones las analiza Gentes con detalle (Gentes, 2002; Gentes, 2004).

\section{Extractivismo, hidroeléctricas e industria forestal}

El extractivismo o su conceptualización contemporánea, neoextractivismo, ha constituido un imperativo en la agenda de los países del Ilamado Sur Global, como una senda productiva para las trasformaciones económicas (Acosta, 2013; Arsel, Hogenboom, \& Pellegrini, 2016). Esta arista del marco neoliberal aplicado en Chile ha facilitado el ingreso de capitales extranjeros en territorios de agricultores, campesinos e indígenas, tal como lo describen las investigaciones de Aitken, Rivera, Godoy-Faúndez y Holzapfel (2016), Jacka (2018) y algunas otras que serán revisadas más adelante. De hecho, algunos estudios establecen que Chile, al compararlo con otros países de similares características de explotación minera, presenta un marco regulatorio que facilita este tipo de actividad económica (Oyarzún \& Oyarzún, 2011; Thomashausen, Maennling, \& Mebratu-Tsegaye, 2018). 
Este tema se sustenta en una literatura desarrollada en la última década, cuyas contribuciones poseen un importante acervo de datos empíricos que aportan a la comprensión del fenómeno del agua en el ámbito minero y, en menor medida, forestal. Así, por ejemplo, Bottaro et al. (2014) han observado en los conflictos contra el proyecto minero Pascua Lama, las formas de la respuesta social a las consecuencias del neoextractivismo en los territorios locales. Acciones similares suceden también con los proyectos hidroeléctricos en la Patagonia, generando luchas socioambientales en las comunidades locales (Romero-Toledo et al., 2009; Torres-Salinas \& García, 2009). En el Biobío es posible observar otro tipo de extractivismo, caso en el que la industria forestal, en un período de 20 años, ha cuadruplicado sus hectáreas de cultivo en desmedro de la agricultura de subsistencia (Torres-Salinas, Gironás, \& Pint, 2016; Torres-Salinas, Azócar, Rojas, Montecinos, \& Paredes, 2015).

Estas disputas son sólo un ámbito de los impactos de un extractivismo que además de depredar los recursos minerales, también lo hace con los hídricos (Bottaro et al., 2014). Al respecto, se ha expuesto que el agua en el desierto de Atacama es escasa, y sus mínimas reservas se ubican en sectores protegidos o con asentamientos ancestrales indígenas. En este territorio se ubican megaproyectos mineros amparados por un aparato estatal que los protege (Bolados-García \& Babidge, 2017). Por ejemplo, la intromisión que amenaza las localidades de la región de Tarapacá produce competencias desiguales por el control de los recursos (Romero-Aravena, Méndez, \& Smith, 2012). En particular, el pueblo atacameño ha sufrido una degradación del ecosistema en la que sus prácticas productivas ancestrales se han visto reducidas 0 , incluso, eliminadas, tal como señalan de forma empírica y diacrónica Prieto, 
Salazar y Valenzuela (2019). En consecuencia, la desposesión produce de manera concomitante configuraciones en el tejido cultural indígena, lo que ha contribuido a resignificar sus prácticas, producto de traslocalizaciones forzadas y a dinámicas de negociación y disputa (Romero-Toledo, 2018). Estos fenómenos estudiados por Romero-Toledo (2018) habían constituido años antes el centro de la investigación etnográfica de Carrasco (2016) en otros casos de estudio, pues Carrasco se interesó por las contradicciones simbólicas y valorativas como corolarios de un territorio altamente mercantilizado. Este escenario fenomenológico permite iluminar las confluencias cosmogónicas de la dualidad del agua para los indígenas: desde la cosmovisión andina, un elemento indisociable de la cultura, y en la actual práctica productiva, una mercancía transable.

Según Babidge (2013), otro de los artificios utilizados por la megaminería corresponde a las acciones con carátula de Responsabilidad Social Empresarial (RSE): medidas dirigidas a presentar el extractivismo con un espíritu sustentable. Los programas de RSE, en efecto, irían en supuesto beneficio de las comunidades afectadas, donde sería posible entrever la formación de un clientelismo o, como Babidge (2016) ilustra, la disímil idea de socios. Más tarde, la misma investigadora también sugiere que una de las estrategias de control de los proyectos mineros es sostener la ignorancia y la confusión como herramienta de control de las comunidades (Babidge, 2019).

Para otros investigadores, los impactos ambientales del neoextractivismo en los grupos humanos son problemas que deberían superarse para hacer posible una minería responsable (Acosta, 2018). Sin embargo, al observar los hallazgos e intereses económicos, pareciera que 
una conservación de los ecosistemas y de las dinámicas culturales ancestrales únicas, en la praxis, no constituye una prioridad para los proyectos de megaminería ni para el Estado (Romero-Aravena et al., 2012). De los problemas categorizados en este tema, también están los conflictos entre comunidades locales y agentes externos, fenómeno que se revisa a continuación.

\section{Conflictos socio-territoriales por el agua}

De acuerdo con De la Maza, Maillet y Martínez-Neira (2017), el agua sería una de las principales razones de los conflictos socio-territoriales en Chile. Un tema de investigación dominado por un foco en las disputas con agentes externos. Así, por ejemplo, Usón, Henríquez y Dame (2017) observan en el caso de la cuenca alta de Yalí, cómo las empresas agroindustriales manejan una tecnicidad de la información que les permite sostener la ignorancia sobre datos hidrogeológicos de la cuenca. Una estrategia que Babidge (2019) pudo pesquisar en contextos de la gran minería. Ambas situaciones ilustran cómo las redes complejas de intereses se enfrentan desde disímiles posiciones de poder vulnerando a las comunidades locales (Usón et al., 2017). De esta forma, los grupos humanos afectados por disputas de agua muestran una politización de sus acciones, expresada a través de los discursos contra agentes extemos que ingresan a devastarel territorio (Romero-Toledo et al., 2009; Torres- 
Salinas et al., 2017). Según Torres-Salinas y Rojas (2018), los movimientos hidrosociales serían una respuesta a la fractura hidrometabólica del neoliberalismo. A la luz de un análisis de redes sociales (ARS) en otro caso de estudio, es de reconocer que estos movimientos de lucha serían clusters con un escaso nivel de interacción entre ellos para una misma acción colectiva (Fonseca, Montalba, \& García, 2015). Sin embargo, mantienen funciones propias para el fortalecimiento de la identidad grupal y sus niveles de cohesión interna (Castillo, 2016; Gentes, 2002; Molina, 2012; Romero-Toledo, Videla, \& Gutiérrez, 2017).

Otros investigadores han puesto la mirada en diferentes tipos de conflictos. Gentes (Gentes, 2000; Gente, 2002; Gentes, 2003; Gentes, 2004) ostenta una larga trayectoria de investigación acerca de los marcos legales y las acciones sistemáticas del Estado por no reconocer el derecho consuetudinario indígena. Estas intersecciones conflictivas derivadas en la inexistencia de un pluralismo legal también fueron investigadas por Castro (1997), Cuadra (2000), y Castro y Quiroz (2011). De estas contribuciones, al respecto, se sostiene que la postergación y el ocultamiento de las prácticas normativas indígenas conllevan una violación del derecho consuetudinario, toda vez que la asimilación cultural es un proceso constante para el progreso neoliberal, tal como para otras corrientes que apuntan a la modernización.

En otro ámbito destino a la privatización del agua, Garande y Dagg (2005), y Cantillana (2018) sostienen que la política chilena sobre el acceso al agua potable de la comunidades rurales tiene como consecuencia la emergencia de conflictos internos. Destacan sobre todo que el Programa de Agua Potable Rural (APR) tiene una imposición top- 
down que además exacerba la marginación de los conocimientos locales de gestión del agua. Cantillana (2018) discute que, a pesar de existir un proceso de participación ciudadana, estas acciones performativas y mediáticas sin capacidad resolutiva exhiben la tendencia a desinformar en lugar de buscar consensos entre la comunidad. Estos estudios empíricos (véase también a Suarez, 2018) contradicen las conclusiones positivas al Programa APR contenidos en la investigación de tipo secundaria de Blanco y Donoso (2016).

\section{Gestión y conocimiento local}

La gestión del agua en el nivel local comprende conocimientos ligados con su uso y manejo. En este tema de investigación existen diversos cúmulos de conocimiento que dependen de las características propias de la actividad productiva: agrarios, pecuarios, cazadores-recolectores de borde mar, etcétera. La tendencia descriptiva de tales fenómenos marcó el inicio de la investigación del agua en Chile. Estas primeras investigaciones, durante las últimas dos décadas del siglo pasado, han entregado un acervo de las distintas prácticas indígenas sudandinas (Aldunate, 1985; Castro, 1992; Castro et al., 1991; Hendriks, 1998; Núñez, 1985; Núñez \& Pourrut, 1995; Van Kessel, 1985). Actividades que, según Young et al. (2009), están en peligro debido a los efectos del cambio climático y a la depredación del agua. En otra contundente 
investigación al respecto, se puede consultar la obra seminal de Yáñez y Molina (2011) titulada: Las aguas indígenas en Chile.

Estos conocimientos son tan sólo una de las múltiples conexiones en la que también se revela la dimensión ritual y simbólica. En la cultura mapuche, por ejemplo, se ha investigado la utilización de modelos dendríticos y reticulares en torno a la percepción de los paisajes del agua (Skewes, Solari, Guerra, \& Jalabert, 2012). El uso del espacio y su relación simbólica también ha sido examinado por la arqueología en torno a los restos materiales del período formativo en la región centro-sur andina (Muñoz-Ovalle, 2013). Para los indígenas aimara, el culto a sus mallkus o achachilas (deidades) constituyen ceremonias rogativas que suplican por la abundancia de agua para las cosechas fértiles (Choque \& Pizarro, 2013). Desde una perspectiva metodológica, Di Giminiani y González (2018), con una mirada a las investigaciones centradas en la relación entre seres humanos y agua, sugieren que una perspectiva de objetivación inacabada sería una forma más precisa de aproximación a un análisis certero de esta problemática.

Otros investigadores de la gestión local se han interesado por las prácticas de campesinos rurales no indígenas. Al observar algunos casos de la región de Valparaíso, es posible entrever cómo la alta demanda de recursos hídricos para la mega producción perturban las actividades productivas autosustentables (Panez-Pinto, Mansilla-Quiñones, \& Moreira-Muñoz, 2018). Efectos similares afectan a otro grupo de pequeños agricultores que han debido migrar su actividad productiva hacia la apicultura, fenómeno que, según el análisis empírico de Trujillo (2017), habría permitido una conservación socialmente inclusiva. De igual 
forma, desde una mirada macro, Delgado, Torres-Gómez, Tironi-Silva y Marín (2015) revisan el informe de adaptación al cambio climático para ilustrar la vulnerabilidad en la que se encuentra la agricultura de subsistencia (véase también a Roco, Poblete, Meza, \& Kerrigan, 2016). Para Hurlbert y Diaz (2013), la adaptación al cambio climático y las acciones de mitigación correspondientes a la degradación ambiental no serían posibles desde la lógica neoliberal y, en absoluto, desde los intereses económicos. En definitiva, la acción gubernamental para la gestión del agua en Chile constituiría un modelo cuestionable para enfrentar los desafíos del cambio climático (Hill \& Allan, 2014).

\section{Sistemas de provisión de agua potable}

Los servicios sanitarios públicos chilenos comenzaron a operar en el año 1931. Este inicio de las instituciones sanitarias estuvo marcado por un tratamiento del agua como bien público, seguido de una etapa de modernización que convergió en una actual fase de privatización, algunas descripciones históricas se pueden encontrar en las producciones de AléYarad (1990), Alegría y Celedon (2006), y Fischer y Serra (2004). Lo que más se destaca de este proceso es la amplia cobertura lograda en áreas urbanas que alcanzó el 98\% (Alegría \& Celedon, 2006). Por otra parte, es importante reconocer que la conceptualización sobre la privatización del agua en Chile (véase también a Morandé y Doña, 1999) se relaciona de 
modo directo con el tratamiento jurídico del CA, que permitió la comercialización privada de los recursos hídricos en un nivel nacional. Dicho panorama no debe ser confundido con el proceso de privatización de las empresas sanitarias del Estado, el cual ostenta características particulares, y que además ha congregado gran atención. De igual forma se suman a la literatura recientemente citada las contribuciones sobre el análisis histórico del acceso y distribución de agua potable (Pflieger, 2008; Simón-Ruiz \& Sánchez Andaur, 2018) y sus sistemas tarifarios (Castro, 2008; Fercovic, Foster, \& Melo, 2019; Molinos-Senante, 2018; Vargas, 2002).

En general, el proceso de privatización de las instituciones sanitarias ha sido valorado por algunos estudios basados en datos estadísticos como un proceso exitoso (e.g., Donoso, 2015b; Molinos-Senante \& SalaGarrido, 2016). Esta valoración ha sido criticada desde el análisis de los sistemas tarifarios de Durán (2015), cuyos resultados permiten revelar la afectación de estas empresas a los procesos de segregación urbana. En otro ámbito, Delgado, Arumí y Reicher (2017) advierten que tampoco existe un cuerpo normativo que obligue a las empresas sanitarias a proteger las captaciones de agua potable de riesgos de contaminación. Menos aun existiría una preocupación normativa por asegurar la continuidad hídrica en contextos de crisis, toda vez que el actual modelo no permite afrontar el binomio de las escasez/sustentabilidad (Lehn et al., 2012; Salinas, Gironás, \& Pinto, 2016). 


\section{Viejos pretextos, nuevos desafíos}

Los investigadores han brindado una próspera y heterogénea literatura: próspera en cuanto a su desarrollo y heterogénea disciplinariamente, en el que las distintas contribuciones revisadas exhiben un examen procesual y multidisciplinar en el abordaje de los fenómenos hídricos. Los intereses, pese a los esfuerzos realizados para su clasificación en temas principales, transitan entre diferentes dominios, revelando las múltiples y diversas intersecciones del agua. A pesar de las características generales de este estado del arte, distintas investigaciones reflejan una carencia empírica en la información entregada. Por ejemplo, las tradiciones de investigación que enaltecen las particularidades del actual sistema normativo y transaccional del agua son las que analizan datos secundarios y estadísticos. Estas producciones, por lo tanto, pueden conllevar abstracciones o elucubraciones no suficientemente contrastadas, permitidas por un trabajo de campo inexistente o limitado. Una impronta que queda expuesta a la luz de la evidencia de investigaciones que, en contra ejemplo, emplean trabajo de campo para la recolección de sus datos. Más de una década después del llamado de Bauer (1997) sobre la necesidad de estudios empíricos para la investigación hídrica, y a pesar de las reconocidas contribuciones que en último tiempo se han realizado, continúa el predominio de investigaciones de corte cuantitativo que, incluso reconociendo su valor, deberían ser complementadas con análisis más integrales de este fenómeno multidimensional. Esta forma para 
superar tal situación debe basarse en aumentar las investigaciones con recolección de datos in situ y con una mirada global, la que surge de este examen a las investigaciones revisadas: el agua no es sólo un elemento físico natural ni se reduce al consumo domiciliario, menos aun exclusivamente un insumo para la producción minera, ni tan sólo parte de un sistema simbólico. En alegoría a estas particularidades se constata la complejidad del fenómeno del agua: en modo alguno, una realidad física separada del tejido sociocultural.

Este tratamiento para la comprensión de los fenómenos representa desafíos que los nuevos enfoques investigativos y modelos teóricos, en parte, consideran. En particular, se observan las propuestas teóricas neomarxistas y de la ecología cultural, transformados hoy en la vanguardia a través de la EP, justicia ambiental, modelo hidrosocial y la fractura socio-metabólica. La integración de estos enfoques ha ayudado a la articulación de las lógicas que subyacen a la crisis hídrica con un análisis desde las dimensiones macroestructurales. A pesar de los avances en esta dirección y al reconocimiento de las contribuciones interdisciplinares, parecen desdibujarse las aportaciones propias que cada disciplina puede aportar al debate. Por lo anterior, se intenta argumentar que la utilización de los enfoques transdisciplinares anteriormente descritos debería, al mismo tiempo, intentar aplicar los enfoques y conocimientos propios de cada disciplina. Este desafío epistemológico no pretende una vuelta al enclaustramiento disciplinaren el que cada una de ellas atienda en sus formas y directrices a su objeto de estudio como cuestiones autárquicas, sino una renovación de un debate interdisciplinario (Max-Neef, 2005). 
En conclusión, quedan en relieve las consecuencias ambientales de la agenda política y del poder económico, demostrando en su totalidad la incapacidad del mercado por aportar a un modelo de equidad, integración y de sostenibilidad social y ambiental. Por otra parte, las configuraciones socioculturales resultantes de este programa de degradación ambiental, así como las nuevas dialécticas entre mujeres, hombres y su entorno socio-ecológico, constituyen un espacio profundo con diversos problemas de conocimiento. Un escenario emocionante para investigaciones futuras que, sin duda, se ampliará en la medida que se oriente cada vez más a la búsqueda empírica de las múltiples conexiones e intersecciones del fenómeno hídrico y su actual contexto de crisis.

\section{Agradecimientos}

Los más sinceros agradecimientos a José Luis Molina e Irene IniestaArandia por las continuas observaciones realizadas a este manuscrito, y también a los evaluadores, cuyas indicaciones permitieron enriquecerlo. De igual forma se agradece al financiamiento N²72190334 PFCHA de la Agencia Nacional de Investigación y Desarrollo de Chile.

\section{Referencias}

Acosta, A. (2013). Extractivism and neoextractivism: Two sides of the same curse. In: Lang, M., \& Mokrani, D. (eds.). Beyond development: Alternative visions from Latin America (Vol. 1, pp. 61-86). Amsterdam, The Netherlands: Transnational Institute.

Acosta, O. (2018). Water and mining. In: Donoso, G. (ed.). Water policy 
Tecnología y

Ciencias đgua
2020, Instituto Mexicano de Tecnología del Agua

Open Access bajo la licencia CC BY-NC-SA 4.0

(https://creativecommons.org/licenses/by-nc-sa/4.0/)

in Chile (pp. 179-193). Recuperado de https://doi.org/10.1007/978-3-319-76702-4

Aitken, D., Rivera, D., Godoy-Faúndez, A., \& Holzapfel, E. (2016). Water scarcity and the impact of the mining and agricultural sectors in Chile. Sustainability, 8(2), 128. Recuperado de https://doi.org/10.3390/su8020128

Aldunate, C. (1985). Desecacion de las Vegas de Turi. Chungara. Revista de Antropología Chilena, (14), 135-139.

Alé-Yarad, J. (1990). Un nuevo esquema de regulación de monopolios naturales. Estudios Públicos, 37, 165-226.

Alegría, M., \& Celedon, E. (2006). Historia del sectorsanitario chileno. De la gestión hasta el proceso de privatización. Zúrich, Suiza: Instituto de Investigación de las Naciones Unidas para el Desarrollo Social (UNRISD).

Arsel, M., Hogenboom, B., \& Pellegrini, L. (2016). The extractive industries and society. The extractive imperative in Latin America. The Extractive Industries and Society, 3(4), 880-887. Recuperado de https://doi.org/10.1016/j.exis.2016.10.014

Babidge, S. (2013). "Socios": The Contested Morality of "Partnerships" in indigenous community-mining company relations, Northern Chile. Journal of Latin American \& Caribbean Anthropology, 18(2), 274293. Recuperado de https://doi.org/10.1111/jlca.12020

Babidge, S. (2016). Contested value and an ethics of resources: Water, mining and indigenous people in the Atacama Desert, Chile. The Australian Journal of Anthropology, 27(1), 84-103. Recuperado de 
Tecnología y

Ciencias $\stackrel{\Xi}{\unlhd}$ gua
2020, Instituto Mexicano de Tecnología del Agua

Open Access bajo la licencia CC BY-NC-SA 4.0

(https://creativecommons.org/licenses/by-nc-sa/4.0/)

https://doi.org/10.1111/taja.12139

Babidge, S. (2019). Sustaining ignorance: The uncertainties of groundwater and its extraction in the Salar de Atacama, northern

Chile. Journal of the Royal Anthropological Institute, 25(1), 83-102.

Recuperado de https://doi.org/10.1111/1467-9655.12965

Babidge, S., \& Bolados, P. (2018). Neoextractivism and indigenous water ritual in Salar de Atacama, Chile. Latin American Perspectives, 45(5), 170-185. Recuperado de https://doi.org/10.1177/0094582X18782673

Baer, M. (2014). Private water, public good: Water privatization and state capacity in Chile. Studies in Comparative International Development, 49(2), 141-167. Recuperado de https://doi.org/10.1007/s12116-014-9154-2

Bauer, C. (1993). Los derechos de agua y el mercado: efectos e implicancias del Código de Aguas chileno de 1981. Actas Del Seminario sobre Política Nacional de Aguas (1991). Revista de Derecho de Aguas, 4, 17-63.

Bauer, C. (1997). Bringing water markets down to earth: The political economy of water rights in Chile, 1976-1995. World Development, 25(5), 639-656.

Bauer, C. (1998). Against the current: Privatization, water markets, and the state in Chile. New York, USA: Kluwer Academic Publishers.

Bauer, C. (2004). Siren song. Chilean water law as a model for international reform. New York, USA: Routledge. Recuperado de https://doi.org/10.4324/9781936331062 
Bauer, C. (2005). In the image of the market: The Chilean model of water resources management. International Journal of Water, 3(2), 146. Recuperado de https://doi.org/10.1504/IJW.2005.007283

Bauer, C. (2008). The experience of Chilean water markets. In: Expo Zaragoza: Water Economics and Financing, 1-11. Zaragoza, España: Oficina Internacional de Exposiciones.

Bauer, C. (2013). The experience of water markets and the market model in Chile. In: Meastu, J., (ed.). Water trading and global water scarcity: International experiences (pp. 130-143). Recuperado de https://doi.org/10.4324/9780203084151

Bauer, C., \& Catalán, L. (2017). Water, law, and development in Chile/California cooperation, 1960-70s. World Development, 90, 184-198. Recuperado de https://doi.org/10.1016/j.worlddev.2016.09.006

Bitran, E., Rivera, P., \& Villena, M. J. (2014). Water management problems in the Copiapó Basin, Chile: Markets, severe scarcity and the regulator. Water Policy, 16(5), 844-863. Recuperado de https://doi.org/10.2166/wp.2014.107

Blanco, E., \& Donoso, G. (2016). Agua potable rural: desafíos para la provisión sustentable del recurso. Actas de Derecho de Aguas, (6), 63-79.

Boelens, R., Gentes, I., Gil, A., \& Urteaga, P. (2005). Special law: Recognition and denial of diversity in andean water control. In: Roth, D., Boelens, R., \& Zwarteveen, M. (eds.). Liquid relations: Contested Water rights and legal complexity (pp. 144-171). New 
Brunswick, USA: Rutgers University Press.

Boelens, R., \& Vos, J. (2012). The danger of naturalizing water policy concepts: Water productivity and efficiency discourses from field irrigation to virtual water trade. Agricultural Water Management, 108, 16-26. Recuperado de https://doi.org/10.1016/j.agwat.2011.06.013

Bolados-García, P., \& Babidge, S. (2017). Ritualidad y extractivismo. La limpia de canales y las disputas por el agua en el salar de atacamanorte de Chile. Estudios Atacameños, (54), 207-216. Recuperado de https://doi.org/10.4067/S0718-10432016005000026

Bottaro, L., Latta, A., \& Sola, M. (2014). La politización del agua en los conflictos por la megaminería: discursos y resistencias en Chile y Argentina. Revista Europea de Estudios Latinoamericanos y del Caribe, (97), 97-115.

Recuperado de https://doi.org/10.2307/23972442

Budds, J. (2004a). Power, nature and neoliberalism: The political ecology of water in Chile. Singapore Journal of Tropical Geography, 25(3), 322-342.

Budds, J. (2009). Contested $\mathrm{H}_{2} \mathrm{O}$ : Science, policy and politics in water resources management in Chile. Geoforum, 40(3), 418-430. Recuperado de https://doi.org/10.1016/j.geoforum.2008.12.008

Budds, J. (2010). Water rights, mining and indigenous groups in Chile's Atacama. In: Boelens, R., Getches, D., \& Guevara, J. (eds.). Out of the mainstream: Water rights, politics and identity (pp. 197-211). London, UK: Earthcan. 
Budds, J. (2013). Water, power, and the production of neoliberalism in Chile, 1973-2005. Environment and Planning D: Society and Space, 31(2), 301-318. Recuperado de https://doi.org/10.1068/d9511

Budds, J. (2018). Securing the market: Water security and the internal contradictions of Chile's Water Code. Geoforum, (January), 1-11. Recuperado de https://doi.org/10.1016/j.geoforum.2018.09.027

Cantillana, R. (2018). Conflictos por el agua en sus dimensiones sociales y culturales: política de Estado y gestión comunitaria en Tarapacá, Chile. Estudios Socioterritoriales. Revista de Geografía, (23), 2743.

Carrasco, A. (2016). A biography of water in Atacama, Chile: Two Indigenous community responses to the extractive encroachments of mining. Journal of Latin American and Caribbean Anthropology, 21(1), 130-150. Recuperado de https://doi.org/10.1111/jlca.12175

Carta de Punta del Este. (1961). Carta de Punta del Este. El Trimestre Económico, 24(4), 735-764.

Castillo, M. (2016). Socio-ecological inequality and water crisis: Views of indigenous communities in the Alto Loa Area. Environmental Justice, 9(1), 9-14. Recuperado de https://doi.org/10.1089/env.2015.0023

Castro, J. P. (2008). Water services in Latin America: Experiences with public private partnerships. International Journal of Water, 4(3/4), 235. Recuperado de https://doi.org/10.1504/ijw.2008.019496

Castro, M. (1992). Cultura hídrica: un caso en Chile. La Habana, Cuba: 
Oficina Regional de Cultura para América Latina y el Caribe (ORCAL).

Castro, M. (1997). Agua, derechos y cultura en los Andes del norte de Chile. Un enfoque desde la antropología jurídica. Chungara. Revista de Antropología Chilena, 29(1), 63-80.

Castro, M., Bahamondes, M., Jaime, M., Meneses, C., \& Navarrete, C. (1991). Cultura, tecnología y uso del agua en un pueblo andino del norte de Chile. Revista Chilena de Antropología, (10), 45-69. Recuperado de https://doi.org/10.5354/0719-1472.1991.17681

Castro, M., \& Quiroz, L. (2011). La crisis del agua en Chile: "El futuro de Chile requiere una nueva política de agua". En: Boelens, R., Cremers, L., \& Zwarteveen, M. (eds.), Justicia hídrica. Acumulación, conflicto y acción social (pp. 225-240). Lima, Perú: Instituto de Estudios Peruanos, Fondo Editorial PUCP.

Celedon, E., \& Alegría, M. (2004). Análisis del proceso de privatización de los sistemas de agua potable y saneamiento urbanos en Chile. Rega, 1(2), 65-85.

Choque, C., \& Pizarro, E. (2013). Identidades, continuidades y rupturas en el culto al agua y a los cerros en Socoroma, una comunidad andina de los Altos de Arica. Estudios Atacameños, (45), 55-74. Recuperado de https://doi.org/10.4067/S071810432013000100005

Congreso Nacional de Chile. (1981). Código de Aguas. Santiago de Chile, Chile: Biblioteca del Congreso Nacional.

Cuadra, M. (2000). Teoría práctica de los derechos ancestrales de agua 
de la comunidades atacameñas. Estudios Atacameños, 19(19), 93112.

Recuperado

de

https://doi.org/10.22199/S07181043.2000.0019.00005

De la Maza, G., Maillet, A., \& Martínez-Neira, C. (2017). Socio-territorial conflicts in Chile: Configuration and politicization (2005-2014). European Review of Latin American and Caribbean Studies, 104(104), 23-46. Recuperado de https://doi.org/10.18352/erlacs.10173

Delgado, L., Torres-Gómez, M., Tironi-Silva, A., \& Marín, V. H. (2015). Estrategia de adaptación local al cambio climático para el acceso equitativo al agua en zonas rurales de Chile. America Latina Hoy, 69 113-137. Recuperado de https://doi.org/10.14201/alh201569113137

Delgado, V., Arumí, J. L., \& Reicher, O. (2017). Problemas que plantea la regulación de las áreas de protección de los derechos de aprovechamiento de las aguas subterráneas en Chile, cuando sirven a captaciones de agua potable. Revista de Derecho, (2), 143180.

Di Giminiani, P., \& González, M. (2018). Who owns the water? The relation as unfinished objectivation in the Mapuche lived world. Anthropological Forum, 28(3), 199-216.

Domper, M. de la L. (2006). La privatización del agua y de las empresas sanitarias en Chile (vol. 173). Santiago de Chile, Chile: Libertad y Desarrollo.

Donoso, G. (2013). The evolution of water markets in Chile. In: Maestu, 
J. (ed.). Water trading and global water scarcity: International experiences (pp. 111-129). Recuperado de https://doi.org/10.4324/9780203084151

Donoso, G. (2015a). Chilean water rights markets as a water allocation mechanism. En: Lago, M., Mysiak, J., Gómez, C., Delacámara, G., \& Maziotis, A. (eds.). Use of economic instruments in water policy: Insights from international experience (pp. 265-278). Recuperado de https://doi.org/10.1007/978-3-319-18287-2_197-2

Donoso, G. (2015b). Water pricing in Chile: Decentralization and market reforms. In: Dinar, A., Pochat, V., \& Albiac-Murillo, J. (eds.). Water princing experiences and innovations (pp. 83-96). Recuperado de https://doi.org/10.1007/978-3-319-16465-6

Durán, G. (2015). Agua y pobreza en Santiago de Chile: morfología de la inequidad en la distribución del consumo domiciliario de agua potable. EURE (Santiago), 41(124), 225-246. Recuperado de https://doi.org/10.4067/S0250-71612015000400011

Fercovic, J., Foster, W., \& Melo, O. (2019). Economic development and residential water consumption in Chile. Environment and Development Economics, 24, 23-46. Recuperado de https://doi.org/10.1017/S1355770X18000463

Fischer, R., \& Serra, P. (2004). Efectos de la privatización de servicios públicos en Chile: casos sanitario, electricidad y telecomunicaciones. Washington, DC, USA: Banco Interamericano de Desarrollo.

Fonseca, F., Montalba, R., \& García, M. (2015). Redes sociales, capital 
social y acción colectiva en dos territorios campesinos de la región de La Araucanía, en Chile, para enfrentar problemas asociados al acceso al agua. Papers. Revista de Sociología, 100(4), 577-606. Recuperado de https://doi.org/10.5565/rev/papers.2169

Garande, T., \& Dagg, S. (2005). Public participation and effective water governance at the local level: A case study from a small underdeveloped area in Chile. Environment, Development and Sustainability, 7(4), 417-431. Recuperado de https://doi.org/10.1007/s10668-004-3323-9

Gazmuri, R. (1992). Chile's market-oriented water policy: Institutional aspects and achievements. In: Le Moigne, G., Easter, K., Ochs, W., \& Giltner, S. (eds.). The World Bank's Ninth Annual Irrigation and Drainage Seminar (pp. 65-78). Maryland, USA: World Bank Technical Paper.

Gentes, I. (2000). Culturas étnicas en conflicto: el código de aguas y las comunidades indígenas en el Norte Grande, Chile. Revista Américas, 16(4), 7-50.

Gentes, I. (2002). Agua, poder y conflicto étnico. Legislación de recursos hídricos y reconocimiento de los derechos indígenas en los países andinos: importancia, obstáculos, perspectivas y estrategias. Santiago de Chile, Chile: Comisión Económica para América Latina y el Caribe (Cepal).

Gentes, I. (2003). Gestión comunitaria de propiedad hídrica y manejo de conflictos. Algunas experiencias andinas. Cadernos PROLAM/USP, 2(2), 125-145. Recuperado ode https://doi.org/10.11606/issn.1676-6288.prolam.2003.83034 
Gentes, I. (2004). Agua, derechos localese indígenas y su interacción con la legislación nacional-estudio de caso de Chile. Santiago de Chile, Chile: Universidad de Wageningen-Comisión Económica para América Latina y el Caribe.

Gentes, I. (2006). Las reformas al código de aguas en Chile: ¿más de lo mismo o cambio de paradigmas en las políticas hídricas? Congreso Internacional WALIR: Pluralismo Legal, Recursos Hídricos y Políticas de Reconocimiento, 175-198. Cusco, Perú.

Grafton, R. Q., Libecap, G., McGlennon, S., Landry, C., \& O’Brien, B. (2011). An integrated assessment of water markets: A crosscountry comparison. Review of Environmental Economics and Policy, 5(2), 219-239. Recuperado de https://doi.org/10.1093/reep/rer002

Guerrero, V. (1991). Recursos hídricos y conflictos sociales en Tarapacá (No. 5). Iquique, Chile: Centro de Investigación de la Realidad del Norte.

Harvey, D. (2005). A brief history of neoliberalism. Oxford, UK: Oxford University Press.

Hearne, R., \& Donoso, G. (2014). Water markets in Chile: Are they meeting needs? In: Easter, K., \& Huang, Q. (eds.). Water markets for the 21st century (pp. 103-126). Recuperado de https://doi.org/10.1007/978-94-017-9081-9

Hendriks, J. (1998). Water as Private property: Notes on the case of Chile. In: Searching for equity: Conceptions of justice and equity in peasant irrigation (pp. 297-309). Assen, The Netherlands: Van 
Tecnología y

Ciencias $\stackrel{\Xi}{\unlhd}$ gua
2020, Instituto Mexicano de Tecnología del Agua

Open Access bajo la licencia CC BY-NC-SA 4.0

(https://creativecommons.org/licenses/by-nc-sa/4.0/)

Gorcum.

Hill, M. (2013). Water governance in the context of IWRM: Chile. In: Hill, M. (ed.). Climate change and watergovernance. Advances in global change research, 54, 141-154. Recuperado de https://doi.org/10.1007/978-94-007-5796-7_8

Hill, M., \& Allan, A. (2014). Adaptive capacity in a chilean context: A questionable model for Iatin america. Environmental Science and Policy, 43, 78-90. Recuperado de https://doi.org/10.1016/j.envsci.2013.10.014

Hurlbert, M., \& Diaz, H. (2013). Water governance in Chile and Canada: A comparison of adaptive characteristics. Ecology and Society, 18(4), 61. Recuperado de https://doi.org/10.5751/ES-06148180461

Hurlbert, M., \& Gupta, J. (2015). The split ladder of participation: A diagnostic, strategic, and evaluation tool to assess when participation is necessary. Environmental Science and Policy, 50, 100-113.

Recuperado de https://doi.org/10.1016/j.envsci.2015.01.011

International Conference on Water and the Environment. (1992). The Dublin statement on water and sustainable development. Recuperado de http://www.un-documents.net/h2o-dub.htm

Jacka, J. (2018). The anthropology of mining: The social and environmental impacts of resource extraction in the mineral age. Annual Review of Anthropology, 47(1), 61-77. Recuperado de https://doi.org/10.1146/annurev-anthro-102317-050156 
Tecnología y

Ciencias $\stackrel{\Xi}{\unlhd}$ gua
2020, Instituto Mexicano de Tecnología del Agua

Open Access bajo la licencia CC BY-NC-SA 4.0

(https://creativecommons.org/licenses/by-nc-sa/4.0/)

Kretshmer, N., Wendt, A., \& Oyarzún, R. (2012). Watershed management: Its scope, a general analysis of its situation in Chile, and one approach to deal with its integrating environmental, political and social issues. In: Rivera, D. (ed.). Chile: Environmental, political \& social issues (pp. 69-90). New York, USA: Nova Science Publishers.

Larraín, S. (2006). El agua en Chile: entre los derechos humanos y las reglas del mercado. Polis. Revista Latinoamericana, (14), s/p.

Lehn, H., Mcphee, J., Vogdt, J., Schleenstein, G., Simon, L., Strauch, G., Nino, Y. (2012). Risks and opportunities for sustainable management of water resources and services in Santiago de Chile. In: Henrichs, D., Martínez, F., Krellenberg, K., \& Hansjürgens, B. (eds.). Risk Habitat Megacity (pp. 251-278). Recuperado de https://doi.org/10.1007/978-3-642-11544-8

Lictevout, E., \& Faysse, N. (2018). A doubly invisible aquifer: Hydrogeological studies and actors' strategies in the Pampa del Tamarugal Aquifer, Northern Chile. Water Alternatives, 11(3), 592606.

Linton, J., \& Budds, J. (2014). The hydrosocial cycle: Defining and mobilizing a relational-dialectical approach to water. Geoforum, 57, 170-180. Recuperado de https://doi.org/10.1016/j.geoforum.2013.10.008

Lovera, D. (2017). Indigenous peoples and the sale of water rights. The case of Chile. In: Russell, A. F. S., \& Langford, M. (eds.). The human right to water: Theory, Practice and prospects (pp. 57-83). Recuperado de https://doi.org/10.1017/9780511862601.005 
Madaleno, I. M., \& Gurovich, A. (2007). Usos conflictivos del agua en el norte de Chile. Boletín de La Asociación de Geógrafos Españoles, (45), 353-372.

Max-Neef, M. (2005). Foundations of transdisciplinarity. Ecological Economics, 53, 5-16. Recuperado de https://doi.org/10.1016/j.ecolecon.2005.01.014

Molina, F. (2012). Competing rationalities in water conflict: Mining and the indigenous community in Chiu Chiu, El Loa Province, Northern Chile. Singapore Journal of Tropical Geography, 33(1), 93-107. Recuperado de https://doi.org/10.1111/j.14679493.2012.00451.x

Molinos-Senante, M. (2018). Urban water management. In: Donoso, G. (ed.). Water Policy in Chile (pp. 131-150). Switzerland, Zurich: Springer International Publishing Switzerland.

Molinos-Senante, M., Donoso, G., \& Sala-Garrido, R. (2016). Are participants in markets for water rights more efficient in the use of water than non-participants? A case study for Limarí Valley (Chile). International Conference on Contaminated Sediments (ContaSed2015) (Vol. 23). Recuperado de https://doi.org/10.1007/s11356016-6187-2

Molinos-Senante, M., \& Sala-garrido, R. (2016). Performance of fully private and concessionary water and sewerage companies: A metafrontier approach. Environmental Science and Pollution Research, 23(12), 11620-11629. Recuperado de https://doi.org/10.1007/s11356-016-6359-0 
Morandé, F., \& Doña, J. (1999). Governance and regulation in Chile: Fragmentation of the public water sector. In: Spiller, P. \& Savedoff, W., (eds.). Spilled water: Institutional commitment in the provision of water services (pp. 177-218). Washington, DC, USA: Inter American Development Bank.

Muñoz-Ovalle, I. (2013). Agua y monumentalidad en el valle de azapa: indicadores del uso del espacio en las poblaciones Alto Ramírez, período formativo, norte de Chile. Chungara. Revista de Antropología Chilena, 44(4), 571-591. Recuperado de https://doi.org/10.4067/s0717-73562012000400003

Núñez, L. (1985). Recuérdalo, aquí estaba el lagar: la expropiación de las aguas del valle de Quisma (I Región). Chungara. Revista de Antropología Chilena, (14), 157-167.

Núñez, L., \& Pourrut, P. (1995). Agua, ocupación del espacio y economía campesina en la región atacameña. Antofagasta, Chile: Universidad Católica del Norte.

Oyarzún, J., \& Oyarzún, R. (2011). Sustainable development threats, inter-sector conflicts and environmental policy requirements in the arid, mining rich, Northern Chile territory. Sustainable Development, 19(4), 263-274. Recuperado de https://doi.org/10.1002/sd.441

Panez-Pinto, A., Mansilla-Quiñones, P., \& Moreira-Muñoz, A. (2018). Agua, tierra y fractura sociometabólica del agronegocio. Actividad frutícola en Petorca, Chile. Bitácora Urbano Territorial, 28(3), 153160. Recuperado de https://doi.org/10.15446/bitacora.v28n3.72210 
Pflieger, G. (2008). Achieving universal access to drinking water and sanitation networks in Santiago de Chile: An historical analysis 1970-1995. Journal of Urban Technology, 15(1), 19-51.

Prieto, M. (2015). Privatizing water in the Chilean Andes: The case of Las Vegas de Chiu-Chiu. Mountain Research and Development, 35(3), 220-229. Recuperado de https://doi.org/10.1659/MRD-JOURNALD-14-00033.1

Prieto, M. (2016). Bringing water markets down to Chile's Atacama Desert. Water International, 41(2), 191-212. Recuperado de https://doi.org/10.1080/02508060.2015.1107400

Prieto, M., Salazar, D., \& Valenzuela, M. (2019). The extractive industries and society. The dispossession of the San Pedro de Inacaliri River: Political ecology, extractivism and archaeology. The Extractive Industries and Society. Recuperado de https://doi.org/10.1016/j.exis.2019.02.004

Ríos, M., \& Quiroz, J. (1995). The market for water rights in Chile: Major issues. World Bank Technical Papers, 285, 1-33.

Roco, L., Poblete, D., Meza, F., \& Kerrigan, G. (2016). Farmers' options to address water scarcity in a changing climate: Case studies from two basins in Mediterranean Chile. Environmental Management, 58(6), 958-971. Recuperado de https://doi.org/10.1007/s00267016-0759-2

Romano, D., \& Leporati, M. (2002). The distributive impact of the water market in Chile: A case study in Limarí Province, 1981-1997. Quarterly Journal of International Agriculture, 41(1-2), 41-58. 
Tecnología y

Ciencias Agua
2020, Instituto Mexicano de Tecnología del Agua

Open Access bajo la licencia CC BY-NC-SA 4.0

(https://creativecommons.org/licenses/by-nc-sa/4.0/)

Romero-Aravena, H., Méndez, M., \& Smith, P. (2012). Mining

Development and Environmental Injustice in the Atacama Desert of Northern Chile. Environmental Justice, 7(2), 70-76. Recuperado de https://doi.org/10.1089/env.2011.0017

Romero-Toledo, H. (2018). Agua, extractivismo y etno-territorialidades: los aymara y los mapuche en Chile. In Agua y disputas territoriales en Chile y Colombia (pp. 57-84). Bogotá: Universidad Nacional de Colombia.

Romero-Toledo, H., Romero-Aravena, H., \& Toledo, X. (2009). Agua, Poder y Discursos: Conflictos Socio-territoriales por la construcción de centrales hidroeléctricas en la Patagonia Chilena. Anuario de Estudios Americanos, 66(2), 81-103. Recuperado de https://doi.org/10.3989/aeamer.2009.v66.i2.318

Romero-Toledo, H., Videla, A., \& Gutiérrez, F. (2017). Explorando conflictos entre comunidades indígenas y la industria minera en Chile: las transformaciones socioambientales de la región de Tarapacá y el caso de Lagunillas. Estudios Atacameños, 55, 231250.

Salinas, C., Gironás, J., \& Pinto, M. (2016). Water security as a challenge for the sustainability of La Serena-Coquimbo conurbation in northern Chile: global perspectives and adaptation. Mitigation and Adaptation Strategies for Global Change, 21(8), 1235-1246. Recuperado de https://doi.org/10.1007/s11027-015-9650-3

Simón-Ruiz, I., \& Sánchez-Andaur, R. (2018). De aguadores ambulantes a empresas distribuidoras. Historia del agua para consumo en Valparaíso (1850-1917). Revista Complutense de Historia de 
Tecnología y

Ciencias $\stackrel{\Xi}{\unlhd}$ gua
2020, Instituto Mexicano de Tecnología del Agua

Open Access bajo la licencia CC BY-NC-SA 4.0

(https://creativecommons.org/licenses/by-nc-sa/4.0/)

América, 44, 191-210. Recuperado de https://doi.org/10.5209/rcha.61085

Skewes, J. C., Solari, M. E., Guerra, D., \& Jalabert, D. (2012). Los paisajes del agua naturaleza e identidad en la cuenca del río Valdivia. Chungara. Revista de Antropología Chilena, 44(2), 299-312. Recuperado de https://doi.org/10.4067/S071773562012000200007

Solanes, M. (2013). The Washington Consensus, Chilean Water Monopolization and the Peruvian Draft Water Law of the 1990s. Water Alternatives, 6(2), 207-217.

Suarez, A. (2018). "At-home ethnography": Insider, outsider and social relations in rural drinking water management in Chile. Journal of Organizational Ethnography, 7(2), 199-211. Recuperado de https://doi.org/10.1108/JOE-12-2017-0072

Thobani, M. (1997). Formal water markets: Why, when, and how to introduce tradable water rights. The World Bank Research Observer, 12(2), 161-179. Recuperado de https://doi.org/10.1093/wbro/12.2.161

Thomashausen, S., Maennling, N., \& Mebratu-Tsegaye, T. (2018). A comparative overview of legal frameworks governing water use and waste water discharge in the mining sector. Resources Policy, 55(July 2017), 143-151. Recuperado de https://doi.org/10.1016/j. resourpol.2017.11.012

Torres-Salinas, R., Azócar, G., Carrasco, N., Zambrano-Bigiarini, M., Costa, T., \& Bolin, B. (2016). Forestry development, waterscarcity, 
and the Mapuche protest for environmental justice in Chile. Ambiente \& Sociedade, 19(1), 121-144. Recuperado de https://doi.org/10.1590/1809-4422asoc150134r1v1912016

Torres-Salinas, R., Azócar, G., Rojas, J., Montecinos, A., \& Paredes, P. (2015). Vulnerability and resistance to neoliberal environmental changes: An assessment of agriculture and forestry in the Biobio region of Chile (1974-2014). Geoforum, 60, 107-122. Recuperado de https://doi.org/10.1016/j.geoforum.2014.12.013

Torres-Salinas, R., \& García, A. (2009). Conflictos por el agua en Chile: el gran capital contra las comunidades locales. Análisis comparativo de las cuencas de los ríos Huasco (desierto de Atacama) y Baker (patagonia austral). Espacio Abierto, 18(4), 695-708.

Torres-Salinas, R., García, A., \& Rojas, J. (2017). Privatizando el agua, produciendo sujetos hídricos: análisis de las políticas de escala en la movilización socio-hídrica contra Pascua Lama e HidroAysén en Chile. Agua y Territorio, (10), 149. Recuperado de https://doi.org/10.17561/at.10.3615

Torres-Salinas, R., \& Rojas, J. (2018). La fractura hidro-metabólica del neoliberalismo: etnografías de la desposesión hídrica en Chile. En: Astrid, U., \& Romero-Toledo, H. (eds.). Agua y disputas territoriales en Chile y Colombia (pp. 147-177). Bogotá. Colombia: Universidad Nacional de Colombia.

Trujillo, F. (2017). La apicultura del valle central chileno como práctica de conservación socialmente inclusiva en un contexto de escasez hídrica socialmente construida. Cultura-Hombre-Sociedad CUHSO, 27(1), 182-204. Recuperado de https://doi.org/10.7770/CUHSO- 
Tecnología y

Ciencias Agua
2020, Instituto Mexicano de Tecnología del Agua

Open Access bajo la licencia CC BY-NC-SA 4.0

(https://creativecommons.org/licenses/by-nc-sa/4.0/)

V27N1-AR

ONU, United Nations. (1977). United Nations Conference on Water. Recuperado de http://www.un.org/es/sections/issuesdepth/water/index.html

Usón, T. J., Henríquez, C., \& Dame, J. (2017). Disputed water: Competing knowledge and power asymmetries in the Yali Alto basin, Chile. Geoforum, 85(July), 247-258. Recuperado de https://doi.org/10.1016/j.geoforum.2017.07.029

Van Kessel, J. (1985). La lucha por el agua en Tarapacá; la visión andina. Chungara. Revista de Antropología Chilena, (14), 141-155.

Vargas, M. (2002). La regulación de las empresas sanitarias en Chile: una revisión crítica. Perspectivas, 6(1), 89-109.

World Water Council. (2018). Vision \& Mission. Recuperado de http://www.worldwatercouncil.org/es/visiones-y-misiones

Yáñez, N., \& Molina, R. (2011). Las aguas indígenas en Chile. Santiago de Chile, Chile: LOM Ediciones.

Young, G., Zavala, H., Wandel, J., Smit, B., Salas, S., Jimenez, E., Feibig, M., Espinoza, R., Diaz, H., Cepeda, J. (2009). Vulnerability and adaptation in a dryland community of the Elqui Valley, Chile. Climatic Change, 98(1-2), 245-276. Recuperado de https://doi.org/10.1007/s10584-009-9665-4 